\title{
Defining ultra artificial intelligence (UAI) implementation using bionic (biological-like- electronics) brain engineering insight
}

\author{
Abstract \\ BIONICS is a common term for bio-inspired information technology, typically including \\ three types of systems, namely: \\ a. bio-morphic (eg: neuromorphic) and bio-inspired electronic/optical devices, \\ b. autonomous artificial sensor-processor-activator prostheses and various devices built \\ into the human body, and \\ c. living-artificial interactive symbioses, e.g. brain-controlled devices or robots. \\ In spite of some restrictive use of the term 'bionics' in popular culture, as well as the \\ unfulfilled promises in the fields of neural networks, artificial intelligence, soft computing \\ and other 'oversold' areas, it was agreed that the name bionics as defined above is the right \\ one for the emergent technology also described as bio-inspired information technology \\ (some people are suggesting info-bionics). There are numerous programs at several funding \\ agencies which are supporting parts of this field under various other names. ${ }^{1}$
}

Volume 2 Issue 2 - 2018

\author{
Sadique Shaikh \\ Institute of Management \& Sciences (IMS), India
}

Correspondence: Sadique Shaikh, Institute of Management \& Sciences (IMS), M.S, India, Email sids_nsk@rediffmail.com

Received: March 12, 2018 | Published: March 26, 2018

\section{Bionic brain}

Bionic Brain stand for "Biological\-like-Electronic" Brain with Mimic Natural Intelligence, Artificially on Silicon Chip which gives similar functioning to Humanoid (Human like Robots) like Biological Brain of human being (Refer my complete paper mentioned in reference to for depth). Scientists are beginning to look much more closely at the mechanisms of the brain and the way it learns, evolves and develops intelligence from a sense of being conscious. For example, AI software designers are beginning to team up with cognitive psychologists and use cognitive science concepts. Another example centers upon the work of the 'connectionists' who draw attention to computer architecture, arguing that the arrangement of most symbolic AI programs is fundamentally incapable of exhibiting the essential characteristics of intelligence to any useful degree. As an alternative, connectionists aim to develop AI t rough artificial neural networks (ANNs). Based on the structure of the nervous system, these 'computational-cognitive models' are designed to exhibit some form of learning and 'common-sense' by drawing links between meanings. ANNs, then, work in a similar fashion to the brain: as information comes in, connections among processing nodes are either strengthened (if the new evidence is consistent) or weakened (if the link seems false).The emergence of ANNs reflects an underlying paradigm change within the AI research community and, as a result, such systems have undeniably received much attention of late. However, regardless of their success in creating interest, the fact remains that ANNs have not nearly been able to replace symbolic AI. As Grosz and Davis (1994) remark: '[Symbolic AI has] produced the technology that underlies the few thousand knowledge-based expert systems used in industry today.' A major challenge for the next decade, then, is to significantly extend this foundation to make possible new kinds of high-impact application systems. A second major challenge will be to ensure that AI continues to integrate with related areas of computing research and other fields. For example, the kinds of developments described for nanotechnology may go some way to accelerating progress in AI, particularly through the sensor interface. ${ }^{2}$ For these reasons, the list of main research areas that follows should be regarded as neither exhaustive nor clear-cut. Indeed, future categorizations will again c

\section{Modelling}

\section{Bionic brain classified engineering (BBCE) model}

This is the first purposed "Bionic Brain Classified Engineering (BBCE)" model through which I would like to focus on major engineering domains of Bionic Brain. The concept exhibit in BBCEModel with classified Bionic Brain engineering with fundamental issue that, what you want to engineer using UAI. Because it very essential need to know though you are design in domain of Bionic Brain, the point here to discuss fundamentally two kinds of Bionic Brain engineering in present research scenario possible, one is "Mimic Bionic Brain" and another is "Born-Child like Bionic Brain". The first one Bionic Brain research in progress in several countries and also in use at initial level in some places, but the second one still need to overcome with several new concepts and engineering ideas to build up domain. I coined terms Mimic Bionic Brain as here AI engineering take place to study some genius intelligence using biological Neuroscience theory and his Natural Intelligence (NI- God made) coped electronically on Chip as Artificial Intelligence (AI-Man made) where as Born-Child like Bionic Brain would be engineering to develop completely blank electronic brain, off course similar like human brain structure but it's not mimic its develop itself just like new born child who scanned environment, develop perception generate meaning and memorize identified all objects with proper communications by all means. I further discussed in my first model most generic engineering skills need to develop such Born-Child like Bionic Brain with discussing hardware's and software's modules requirements as for Mimic Bionic Brain are Advanced Machine Learning System \& Mimic ANN (Artificial Neural Network) Schema 
Engineering at Hardware side and Advanced Artificial Intelligence Programs \& Logic Program modules fit to Neural Schema on Software's side. The Born-Child like Bionic Brain also need same factors but with extension as Advanced Self Machine Learning System \& Blank to self develops ANN Schema Engineering at Hardware's side whereas Ultra Artificial Intelligence (UAI) programs \& Logic Program modules search to fit Neural Schemas at software's side. In hardware and software sides in both bionic brain in all four quadrants again vast subject to engineering (Figure 1).

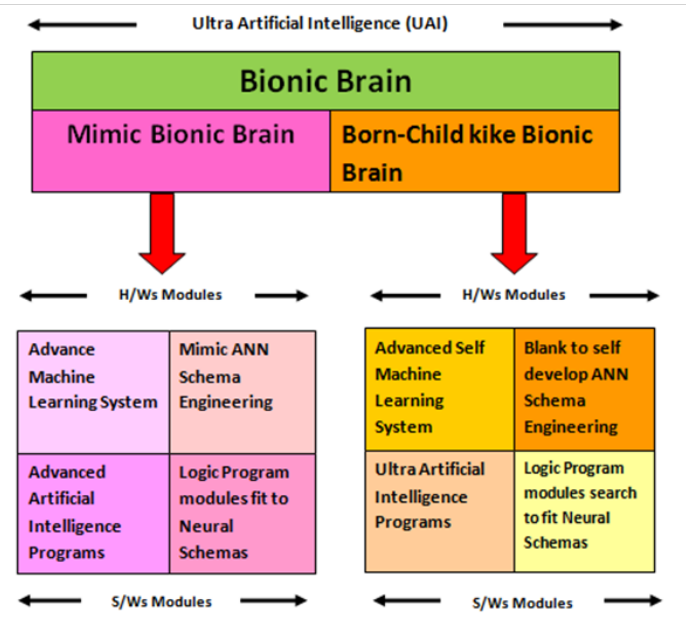

Figure I Ultra artificial intelligence.

Source: Prof. Md. Sadique Shaikh.

\section{Bionic brain engineering insight $(\mathrm{BBCl})$ model}

Figure 2 this my second designed model labeled Bionic Brain Engineering Insight (BBEI) Model which gives you rapid idea how Bionic Brain Engineer, what factors and criterions must consider while engineering and how that engineering will work in integration. This model is completely based on first one with functional engineering description in details. Hence name given Engineering Insight, here complete model split into two segments one is "Cognitive" and another is "Action" having seven layers L1 to L7 for both. In working we can assume same human brain functioning analogically to understand idea. After senses, cognition of environmental stimuli at layer L1 i.e. Cognitive Interface in electronic volume using sensors and transducers its fed to L2 Sensation Handling Unit to filter and channelized from where pass to Sensation Processing to clarify things, elements, visuals, sounds and objects by Biological-Like-Electronic (Bionic) Brain at L3 and send to Alternate Meaning Chamber to acquire meanings at L4. From L4 its passes through L5 Interpreter Unit to make things .i.e. information to intelligence conversion and this input to assembly Memorization often called store/restoration at L6 with attachment L7 Long-Term/Short term memories for different memorization purpose. Whole process begins from top to bottom with L1 to L7. Where as in reverse at L1 Retrieve/Solutions of Interpreter Chamber which make up and concise intelligence for action taking and output performance from Bionic Brain fed to L2 Action Processing Unit to put it in ready state from where goes Action Logic Unit layer L3 where performance strategies and scheduling of action with time control done. The assembly further input to L4 Action Interfacing
Unit to prepare and ready signals for Humanoid Actuators to come in movement according to command and for this L5 \& L6 Actuation Processing and Actuation Handling Unit works together from where all mechanism given to Performance/Action Control \& Output at L7 to out Human-Like performance/action by Humanoid using Bionic Brain Buildup Ultra Artificial Intelligence.

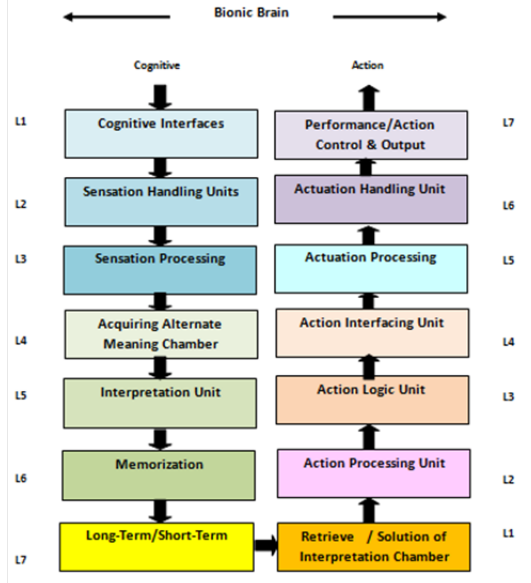

Figure 2 Bionic brain.

Source: Prof. Md. Sadique Shaikh.

\section{Conclusion}

With closing this short communication I would like to conclude "Bionic Brain" is the future of Artificial Intelligence at peak level I coined term "Ultra Artificial Intelligence" here for the same. Using Bionic Brain not only possibilities for human-like/Humanoid Robots, but also Medical Robots, Space Robots for precise space research. One day Bionic Brain and Biological Brain both become tough on each other. The second challenge after this how emotion, feelings, body language, gesture, posture and expression possible to program and control using Bionic Brain in Humanoid. At this stage there is also possibility Robotic Violence with Mankind. I have shown with help of two models how one can start journey towards it.

\section{Acknowledgments}

None.

\section{Conflict of interest}

The authors declare that there is no conflict of interest.

\section{References}

1. NSF/EC Understanding on Co-operation in Information Technologies Strategic Research Workshops, IST 1999-12077.

2. Sadique Shaikh. Analysis and modeling of Strong A.I to engineer BIONIC brain for humanoid robotics application. American Journal of Embedded System and Applications. 2013;1(2):27-36. 